\title{
Peruvian Agricultural Research
}

\author{
ISSNe 2706-9397
}

Homepage: http://revistas.unjfsc.edu.pe/index.php/PeruvianAgriculturalResearch CUniversidad Nacional José Faustino Sánchez Carrión, Lima, Perú

Recibido: Setiembre 2, 2020 / Aceptado: Octubre 19, 2020

2(2), 48-53, 2020

\section{Prevalencia y factores relacionados a la presentación de Fasciola hepatica en bovinos de Huancabamba, Piura, Perú}

\section{Prevalence and factors related to the presentation of Fasciola hepatica in cattle from Huancabamba, Piura, Peru}

\section{R. M. Alva ${ }^{1,2} \mathbb{D}$, J. C. Leiva $^{1} \mathbb{D}$, G. Y. Acuña ${ }^{1}$}

DOI: https://doi.org/10.51431/par.v2i2.641

\section{Resumen}

Objetivos: Determinar la prevalencia y los factores que influyen en la presentación de Fasciola hepatica en bovinos de Huancabamba (Piura, Perú), durante el periodo junio a noviembre del 2018. Metodología: Se analizaron 265 muestras de heces de una población de 860 bovinos, pertenecientes a la asociación ganadera de Huancabamba, criados extensivamente. Se utilizó el método de Dennis para identificar huevos de $F$. hepatica en heces y la prueba de Chi cuadrado para determinar asociación entre los factores de estudio lugar de procedencia, altitud, edad y raza con la presentación de la enfermedad. Resultados: La prevalencia de $F$. hepatica fue de $64,91 \%$. La edad, raza y lugar de procedencia no estuvieron asociados con la presentación de la enfermedad $(P>0,05)$ pero sí la altitud $(P<0,05)$. Conclusiones: La prevalencia de $F$. hepatica en la provincia de Huancabamba fue elevada y se incrementó en relación a años anteriores.

Palabras clave: Prevalencia, Fasciola hepatica, lugar, edad, raza, altitud

\begin{abstract}
Objectives: To determine the prevalence and factors that influence the presentation of Fasciola hepatica in cattle from Huancabamba (Piura, Peru), during the period June to November 2018. Methodology: Two hundred and sixty-five stool samples from a population of 860 animals belonging to the Huancabamba cattle association, raised extensively, were analyzed. The Dennis method was used to identify F. hepatica eggs in feces and the Chi-square test to determine the association between the study factors, place of origin, altitude floor, age and race with the presentation of the disease. Results: The prevalence of $F$. hepatica was $64.91 \%$. Age, race and place of origin were not associated with the presentation of the disease $(P>0.05)$ but the altitude level was $(P<0.05)$. Conclusions: The prevalence of $F$. hepatica in the province of Huancabamba was high and increased in relation to previous years.
\end{abstract}

Keywords: Prevalence, Fasciola hepatica, place, age, race, altitude

\footnotetext{
${ }^{1}$ Departamento de Ciencias Veterinarias, Facultad de Medicina Veterinaria, Universidad Nacional Pedro Ruíz Gallo, Lambayeque, Perú.

${ }^{2}$ Autor para correspondencia: ralva@unprg.edu.pe 


\section{Introducción}

La Distomatosis hepática es una enfermedad parasitaria cuyo agente causal es un tremátode denominado Fasciola hepatica. Esta enfermedad tiene distribución mundial y ocasiona pérdidas estimadas en 2000 millones de dólares (Mungube et al., 2006). Es considerada como una de las enfermedades parasitarias más importantes de los rumiantes domésticos y afecta de manera accidental a herbívoros no rumiantes, omnívoros e inclusive al hombre, por esta razón se le clasifica cómo una enfermedad zoonótica (MasComa et al., 2005; Urquhart et al., 2001).

La $F$. hepatica tiene un ciclo de vida indirecto, necesita de un caracol de la familia Lymnaeidae (géneros Lymnaea, Pseudosuccinea y Fossaria) que es el hospedero intermediario. El parásito adulto se localiza en los conductos biliares del hígado en el hospedero definitivo, donde produce huevos que son evacuados a través del conducto colédoco al intestino, luego se eliminan al exterior con las heces. En el medio ambiente, bajo condiciones adecuadas de temperatura y humedad, los huevos desarrollan y liberan embriones ciliados llamados miracidios que se introducen al caracol y se transforman sucesivamente en los estadios larvales de esporocisto, redia y cercaría, éste última larva abandona el caracol y se adhiere a la vegetación circundante, donde pierden su cola y se enquistan transformándose en metacercaria, que constituyen las forma infectiva (Bethan et al., 2019).

La $F$. hepatica durante su migración por el parénquima hacia los conductos biliares destruye el tejido hepático, ocasionando en el animal parasitado trastornos que afectan la nutrición y se manifiestan con una disminución del apetito, producción de leche y tasa de concepción (Mehmood et al., 2017).

En Perú, la fascioliasis animal está ampliamente distribuida en 21 de las 24 regiones; en el 2005 , el $24,18 \%$ de hígados fueron decomisados en los camales por estar infestados con F. hepatica, siendo Cajamarca, Ancash, La Libertad y Huánuco las regiones que presentan las mayores tasas de hígados decomisados. En las regiones amazónicas de Madre de Dios, Ucayali y Loreto se reportan de manera esporádica, por las condiciones climáticas y ecológicas que no favorecerían el ciclo de vida del parásito (Espinoza et al., 2010). Las pérdidas económicas se estiman en no menos de 50 millones de dólares por año a causa de la mortalidad, disminución de la producción de leche, carne, abortos y alto decomiso de vísceras infectadas (Espinoza et al., 2010) lo que permite colocar a esta parasitosis como la segunda enfermedad parasitaria económicamente importante en la ganadería nacional (Cordero, 2016).

La asociación ganadera de la provincia de Huancabamba posee bovinos de diversas razas y cruces orientados al rubro de producción de leche y carne. La F. hepatica es endémica en la zona y afecta la productividad del ganado vacuno; en el 2015 la prevalencia de esta enfermedad en esta provincia se estimó en 42\% (Quiroz \& Rentería, 2017). No se cuenta con estudios relacionados sobre los factores de riesgo que influyen en la presentación de esta enfermedad en esta provincia, que contribuya a un mejor conocimiento de la enfermedad, para implementar medidas sanitarias estratégicas a fin de reducir su impacto económico en la ganadería de la zona.

\section{Metodología}

El estudio se realizó en el ganado bovino perteneciente a la asociación ganadera de la provincia de Huancabamba, departamento de Piura, durante los meses de junio a noviembre de 2018.

La provincia de Huancabamba es una de las ocho provincias que conforman el departamento de Piura; tiene una extensión total de 4 254,14 $\mathrm{km}^{2}$, cuenta con ocho distritos. Presenta un clima templado, árido y con amplitud térmica moderada, la media anual de temperatura máxima y mínima es $24,1^{\circ} \mathrm{C}$ y $14,3^{\circ} \mathrm{C}$, respectivamente y la precipitación media acumulada anual en el 2015 fue de 476,1 mm (Servicio Nacional de Meteorología e Hidrología, 2018).

La asociación ganadera cuenta con 55 socios que se dedican a la crianza de ganado bovino de las razas Holstein, Brown Swiss, Fleckvieh y criollas. El sistema de crianza es extensivo.

De un total de 860 bovinos se seleccionaron 
265 cabezas $(30,8 \%)$ mediante un muestreo estratificado, teniendo en consideración la edad, raza, lugar de procedencia y altitud. Se recolectaron 100 gramos de heces en una bolsa de plástico, directamente del recto de cada animal. Todas las muestras se acondicionaron en una caja tecnopor con gel refrigerante y se trasladaron inmediatamente al laboratorio de Parasitología Veterinaria de la facultad de Medicina Veterinaria de la Universidad Nacional Pedro Ruiz Gallo, Lambayeque.

El procesamiento de las heces para identificar huevos de $F$. hepatica se realizó siguiendo el procedimiento de Dennis (Dennis et al., 1954). Se consideró como animal positivo cuando se observó un huevo de F. hepatica en la muestra.

La prevalencia $(\mathrm{P})$ se determinó con la siguiente fórmula:

$$
\mathrm{P}=\frac{\mathrm{N}^{\circ} \text { muestras fecales positivas }}{\text { Total de muestras }} \times 100
$$

Se hallaron los intervalos de confianza al 95\% para cada valor de prevalencia, según edad, raza y piso altitudinal. La prueba de Chi cuadrado se utilizó determinar la asociación entre la edad, raza, piso altitudinal y lugar de procedencia con los casos positivos de $F$. hepatica. Para el análisis de los datos se utilizó el programa estadístico Minitab v.19.

\section{Resultados y discusión}

En la Tabla 1 se observa que de 265 muestras 172 resultaron positivas al análisis coproparasitológico, lo que representa una prevalencia de $64.91 \%$. La prevalencia de $F$. hepatica en nuestro país es diversa, como lo demuestran los estudios realizados por Quiroz \& Rentería (2017) en Huancabamba-Piura, 42,50\%; Ticona et al. (2010) en VilcashuamánAyacucho, 47,6\%; Calcina (2015) en MelgarPuno, 60,83\% y Ortiz (2011) en Cajamarca, $80 \%$; similar variación de la prevalencia se observa en otros países de Latinoamérica, entre $16,9 \%$ a $96,5 \%$ (Becerra, 2001). La prevalencia elevada obtenida en el presente estudio se debería a las condiciones de temperatura ambiental y precipitación pluvial de la zona, que favorecen el desarrollo del hospedero intermediario y las formas larvarias del parásito (Luzón-Peña et al., 1995; Cordero del Campillo et al., 1999; Urquhart et al., 2001; Olsen et al., 2015; LópezVillacís et al., 2017).

Tabla 1

Prevalencia de F. hepatica en heces de bovinos de la provincia de Huancabamba, Piura

\begin{tabular}{cccc}
\hline & $\begin{array}{c}\text { Casos } \\
\text { positivos }\end{array}$ & $\begin{array}{c}\text { Prevalencia } \\
(\%)\end{array}$ & $\begin{array}{c}\text { IC } \\
(95 \%)^{1}\end{array}$ \\
\hline Positivo & 172 & 64,91 & $(59,16 ; 70,65)$ \\
Negativo & 93 & 35,09 & $(29,35 ; 40,84)$ \\
\hline Total & 265 & 100 & \\
\hline &
\end{tabular}

La Tabla 2 presenta la prevalencia de $F$. hepatica en relación a la edad, raza, distrito y piso altitudinal. No se determinó asociación $(P>0,05)$ entre la prevalencia de $F$. hepatica con la edad. Resultados similares fueron obtenidos por Paucar et al. (2010) y Calcina (2015) y; no coinciden con los obtenidos por Livia-Córdova et al (2021), Pacheco (2017) y Sanchís et al. (2015) en Uruguay y Lugo et al. (2018) en Venezuela, quienes encontraron una menor prevalencia $(P<0,05)$ en bovinos menores de 02 años en comparación a los adultos. La prevalencia de $F$. hepatica similar obtenido en los grupos etarios se debería a la metodología empleada; en el estudio los rangos de edad fueron bastante espaciados (cada dos años), mientras que en las evaluaciones donde se encontró asociación los rangos de edad fueron más estrechos (6 a 12 meses). En los animales jóvenes el sistema inmunológico aún no está totalmente desarrollado y la respuesta inmune es menos efectiva contra los parásitos que contra las bacterias y virus, debido a que los parásitos poseen una serie de mecanismos para eludir o manipular la inmunidad (Barriga, 1995).

No se encontraron diferencias $(P>0,05)$ entre la presentación de $F$. hepatica y las razas de bovino. Estos resultados difieren de los obtenidos por Sanchís et al. (2015) en Uruguay, quienes encontraron una mayor prevalencia en el ganado lechero de raza Holstein en comparación a las razas cárnicas como el Angus, Hereford y sus cruces; los autores explican esta mayor Peruvian Agricultural Research 2(2), 48-53, 2020 
prevalencia por el sistema de crianza, los bovinos de carne se crían bajo un sistema extensivo, teniendo una mayor posibilidad de entrar en contacto con la forma infectiva del parásito.

No se determinó una asociación significativa $(P>0,05)$ entre la presentación de $F$. hepatica y los distritos de procedencia del ganado. Estos resultados no coinciden con los obtenidos por Calcina (2015), Ticona et al. (2010), LiviaCórdova et al. (2021) y Sanchís et al. (2015) quienes encontraron asociación entre ambas variables. Al parecer, el tamaño muestral reducido en algunos distritos influyó en los resultados. Sin embargo, se encontró asociación $(P<0,05)$ entre la presentación de $F$. hepatica con la altitud. Resultado similar fue obtenido por
Lugo et al. (2018) quien determinó que a mayor altitud existe un mayor riego de infestación. En el estudio se observó una mayor presentación en los pisos altitudinales ubicados entre $\operatorname{los} 1500$ a 2000 y 2500 a $3000 \mathrm{msnm}$, mientras que en los pisos 2000 a 2500 y 3000 a 3500 msnm hubo una menor prevalencia. Además de la altitud, las condiciones climáticas existentes en cada nivel altitudinal afectan en mayor o menor medida la supervivencia y actividad de los hospedadores intermediarios de $F$. hepatica, así como la viabilidad de las metacercarias (Luzón-Peña et al., 1995) y son las que determinan una mayor o menor presentación de la enfermedad (Olsen et al., 2015).

\section{Tabla 2}

Prevalencia según factores asociados a la presencia de Fasciola hepatica en heces de bovinos de la provincia de Huancabamba, Piura

\begin{tabular}{|c|c|c|c|c|c|c|}
\hline & Categoría & $\mathrm{N}^{0}$ & $\begin{array}{c}\text { Casos } \\
\text { positivos }\end{array}$ & $\begin{array}{l}\text { Prevalencia } \\
(\%)\end{array}$ & $\begin{array}{c}\text { IC } \\
(95 \%)^{1}\end{array}$ & Probabilidad \\
\hline \multirow{3}{*}{$\begin{array}{l}\text { Edad } \\
\text { (años) }\end{array}$} & $2-4$ & 141 & 90 & 63,83 & $(55,90 ; 71,76)$ & \multirow{3}{*}{0,935} \\
\hline & $5-7$ & 89 & 60 & 67,42 & $(57,68 ; 77,15)$ & \\
\hline & $8-10$ & 35 & 22 & 62,86 & $(46,85 ; 78,86)$ & \\
\hline \multirow{4}{*}{ Raza } & Holstein & 75 & 49 & 65,33 & $(54,56 ; 76,10)$ & \multirow{4}{*}{0,965} \\
\hline & Brown Swiss & 23 & 14 & 60,87 & $(40,92 ; 80,81)$ & \\
\hline & Criolla & 145 & 94 & 64,83 & $(57,06 ; 72,60)$ & \\
\hline & Otros & 22 & 15 & 68,18 & $(48,72 ; 87,64)$ & \\
\hline \multirow{4}{*}{$\begin{array}{l}\text { Altitud } \\
(\mathrm{msnm})\end{array}$} & {$[1,500-2,000>$} & 17 & 14 & 82,35 & $(64,23 ; 99,99)$ & \multirow{4}{*}{0,001} \\
\hline & {$[2,000-2,500>$} & 149 & 82 & 55,03 & $(47,05 ; 63,02)$ & \\
\hline & {$[2,500-3,000>$} & 60 & 50 & 83,33 & $(73,90 ; 92,76)$ & \\
\hline & {$[3,000-3,500]$} & 39 & 26 & 66,67 & $(51,87 ; 81,46)$ & \\
\hline \multirow{5}{*}{$\begin{array}{l}\text { Lugar } \\
\text { (distrito) }\end{array}$} & Huancabamba & 220 & 146 & 66,36 & $(59,70 ; 7258)$ & \multirow{5}{*}{0,818} \\
\hline & Carmen de la Frontera & 5 & 3 & 60,00 & $(14,66 ; 94,73)$ & \\
\hline & Sondor & 25 & 15 & 60,00 & $(38,67 ; 78,87)$ & \\
\hline & Huarmaca & 5 & 3 & 60,00 & $(14,66 ; 94,73)$ & \\
\hline & Otros & 10 & 5 & 50,00 & $(18,71 ; 8129)$ & \\
\hline
\end{tabular}

${ }^{1} \mathrm{IC}(95 \%)$ : Intervalo de confianza al 95\%. 


\section{Conclusiones}

La prevalencia de $F$. hepatica en bovinos de la asociación ganadera de la provincia de Huancabamba, Piura fue elevada $(64,91 \%)$ y se incrementó en relación a años anteriores. Sólo el nivel de altitud se asoció a la presentación de la enfermedad.

\section{Referencias}

Barriga, O. O. (1995). Avances recientes en inmunología parasitaria. Revista Peruana de Parasitología, 11(1), 76-79. https://sisbib. unmsm.edu.pe/bvrevistas/parasitologia/v11_n1/ Contenido.htm

Becerra, W. M. (2001). Consideraciones sobre estrategias sostenibles para el control de Fasciola hepática en Latinoamérica. Revista Colombiana de Ciencias Pecuarias, 14(1), 2835. https://revistas.udea.edu.co/index.php/rcep/ article/view/323749

Bethan C. J., Davies, D. R., Williams, D. J. L. \& Hodgkinson, J. E. (2019). A review of our current understanding of parasite survival in silage and stored forages, with a focus on Fasciola hepatica metacercariae. Grass and Forage Science, 74(2), 211-217. https://doi. org/10.1111/gfs.12429

Calcina, F. E. (2015). Prevalencia y grado de conocimiento de fascioliosis crónica en vacunos de comunidades del distrito de Santa Rosa, Melgar Puno [tesis de pregrado, Universidad Nacional delAltiplano]. Repositorio Institucional UNAP. http://repositorio.unap.edu.pe/bitstream/ handle/UNAP/1852/Calcina_Murillo_Frank Eduardo.pdf? sequence $=1 \&$ isAllowed $=y$

Cordero del Campillo, M., Rojo, F. A., Martínez, A. R., ; Sánchez, M. C., Hernández, S., Navarrete, I., Diez, P., Quiroz, H. \& Carvalho M. (1999). Parasitologia Veterinaria (2. ${ }^{\circ}$ ed.). McGrawHill Interamericana S.A.

Cordero, K. F. (2016). Prevalencia de Fasciola hepática en bovinos beneficiados en el centro de faenamiento FRISILAC durante los años 20122015 [tesis de pregrado, Universidad Ricardo Palma] Repositorio Institucional URP.

https://repositorio.urp.edu.pe/ bitstream/handle/urp/902/Cordero KF.pdf? sequence $=1 \&$ isAllowed $=\mathrm{y}$

Espinoza, J. R., Terashima, A., Herrera-Velit, P. \& Marcos, L. A. (2010). Fasciolosis humana y animal en el Perú: impacto en la economía de las zonas endémicas. Revista Peruana de Medicina Experimental y Salud Pública 27(4), 604-612. http://www.scielo.org.pe/scielo.php?script=sci arttext\&pid=S1726-46342010000400018\&lng $=$ es\&tlng=es.

Livia-Córdova, G. N., Burga-Cisterna, C. A., Quiroz-Dávila, A., Rentería-Samamé, B., Mercado-Gamarra, A., Del Solar-Vela, M. \& Cárdenas-Callirgos, J. (2021). Prevalencia y factores de riego asociados a la infección por Fasciola hepatica en bovinos de comunidades campesinas de Huancabamba (Piura-Perú). Revista de Investigaciones Veterinarias del Perú, 32(1), e19510. https://doi.org/10.15381/ rivep.v32i1.19510

López-Villacís, I. C., Artieda-Rojas, J. R., MeraAndrade, R. I., Muñoz-Espinoza M. S., RiveraGuerra, V. E., Cuadrado-Guevara, A. C., Zurita-Vásquez, J. H. \& Montero-Recalde, M. A. (2017). Fasciola hepática: relevant aspects in animal health. Journal of the Selva Andina Animal Science, 4(2), 137-146. http://www. scielo.org.bo/pdf/jsaas/v4n2/v4n2_a06.pdf

Lugo, M., Morán, R., Chacín, E., Molina, R., González, L. \& Angulo-Cubillán, F. (2018). Fasciola hepatica (Tremátoda: fasciolidae), en bovinos ubicados en dos niveles altitudinales del estado Mérida, Venezuela. Revista Científica, FCV-LUZ, 28(4), 265-269. http://www.saber. ula.ve/handle/123456789/45355

Luzón-Peña, M., Rojo-Vázquez, F. A., \& GómezBautista, M. (1995). Seasonal availability of Fasciola hepatica metacercariae in a temperate Mediterranean area (Madrid, Spain). Journal of Veterinary Medicine, Serie B, 42(10), 577585. https://doi.org/10.1111/j.1439-0450.1995. tb00751.x

Mas-Coma, S., Bargues, M. D. \& Valero M. A. (2005). Fascioliasis and other plant-borne trematode zoonoses. International Journal for Parasitology, 35(11-12), 1255-1278. https://doi. org/10.1016/j.ijpara.2005.07.010

Mehmood, K., Zhang, H., Sabir, A. J., Abbas, R. Z., Ijaz, M., Durrani, A. Z., Saleem, M. H., Ur Rehman, M., Iqbal, M. K., Wang, Y., Ahmad, H. I., Abbas, T., Hussain, R., Ghori, M. T., Ali, S., Khan, A. U. \& Li, J. (2017). A review on epidemiology, global prevalence and economical losses of fasciolosis in ruminants. Microbial Pathogenesis, 109, 253-262. https:// doi.org/10.1016/j.micpath.2017.06.006

Mungube, E. O., Bauni, S. M., Tenhagen, B. A., Wamae, L. W., Nginy, J. M. \& Mugambi, J. M. (2006). The prevalence and economic significance of Fasciola gigantica and Stilesia hepatica in slaughtered animals in the semiarid coastal Kenya. Tropical Animal Health and Production, 38, 475-483. https://doi. 
org/10.1007/s11250-006-4394-4

Olsen, A., Frankena, K., Bødker, R., Toft, N., Thamsborg, S. M., Enemark, H. L. \& Halasa, T. (2015). Prevalence, risk factors and spatial analysis of liver fluke infections in Danish cattle herds. Parasites \& Vectors, 8,(160),1-10. https:// doi.org/10.1186/s13071-015-0773-x

Ortiz, P. (2011). Estado actual de la infección por Fasciola hepatica en Cajamarca, Perú. Biomédica, 31(sup.3), 172-173. https:// revistabiomedica.org/index.php/biomedica/ article/view/549/678

Paucar, S., Chávez, A., Casas, E., \& Suárez, F. (2010). Prevalencia de Fascioliasis y Paramfistomiasis en el ganado lechero de Oxapampa, Pasco. Revista de Investigaciones Veterinarias del Perú, 21(1):87-92. http://www.scielo.org.pe/ pdf/rivep/v21n1/a13v21n1.pdf

Pacheco, S. M. (2017). Prevalenciay factores de riesgo asociado a la Fasciola hepatica en bovinos [tesis de pregrado, Universidad Politécnica Salesiana]. Repositorio Institucional UPS. http://dspace. ups.edu.ec/handle/123456789/14556

Quiroz, A. \& Rentería, B. (2017). Prevalencia de distomatosis hepática, Coccidiosis y Nematodiosis gastrointestinal en ganado vacuno de los distritos de Huancabamba, Cármen de la Frontera, Sondor y Sondorillo de la provincia de Huancabamba, departamento de Piura - 2016 [tesis de pregrado, no publicada, Universidad Nacional Pedro Ruiz Gallo].

Sanchís, J., Hillyer, G. V., Madeira de Carvalho, L. M., Macchi, M. I., Gomes, C., Maldini, G., Stilwell, G., Venzal, J. M., Paz-Silva, A.,
Sánchez-Andrade, R. \& Arias, M. S. (2015). Riesgo de exposición a Fasciola hepática en ganado vacuno en extensivo de Uruguay y Portugal determinado mediante ELISA y un antígeno recombinante. Archivos de Medicina Veterinaria, 47(2), 201-208. https://dx.doi. org/10.4067/S0301-732X2015000200011

Servicio Nacional de Metereologia e Hidrología. (2018). Datos Estación Metereologica de Huancabamba, Piura. https://www.senamhi. gob.pe/?\&p=estaciones

Ticona, D., Chávez, A., Casas, G., Chavera, A., \& Li, O. (2010). Prevalencia de Fasciola hepatica en bovinos y ovinos de Vilcashuamán, Ayacucho. Revista de Investigaciones Veterinarias del Perú, 21(2): 168-174. http://www.scielo.org.pe/ pdf/rivep/v21n2/a04v21n2.pdf

Urquhart, G. M., Armour, J., Duncan, J. L., Dunn, A. M. \& Yennings, F. W. (2001). Parasitologia Veterinaria (2. ${ }^{\circ}$ ed.). Acribia. 